\title{
The effects of whole body vibration in patients with type 2 diabetes: a systematic review and meta-analysis of randomized controlled trials
}

\author{
Caroline C. Robinson ${ }^{1,2}$, Rodrigo P. G. Barreto ${ }^{1,3}$, Graciele Sbruzzi ${ }^{4}$, \\ Rodrigo D. M. Plentz ${ }^{1,3}$
}

\begin{abstract}
Background: Whole body vibration (WBV) has been used to increase physical activity levels in patients with type 2 diabetes mellitus (T2DM). Objective: To carry out a systematic review of the effects of WBV on the glycemic control, cardiovascular risk factors, and physical and functional capacity of patients with T2DM. Method: MEDLINE, LILACS, PEDro, and Cochrane Central Register of Controlled Trials were searched up to June $1^{\text {st }}$, 2015. Randomized controlled trials investigating the effects of WBV, compared to control or other intervention, on blood glucose levels, blood and physical cardiovascular risk factors, and physical and functional capacity in adult individuals with T2DM. Two independent reviewers extracted the data regarding authors, year of publication, number of participants, gender, age, WBV parameters and description of intervention, type of comparison, and mean and standard deviation of pre and post assessments. Results: Out of 585 potentially eligible articles, two studies (reported in four manuscripts) were considered eligible. WBV interventions provided a significant reduction of $25.7 \mathrm{ml} / \mathrm{dl}$ (95\% CI:-45.3 to -6.1 ; I $\mathrm{I}^{2}$ : $19 \%$ ) in 12 hours fasting blood glucose compared with no intervention. Improvements in glycated hemoglobin, cardiovascular risk factors, and physical and functional capacity were found only at 12 weeks after WBV intervention in comparison with no intervention. Conclusion: WBV combined with exercise seems to improve glycemic control slightly in patients with T2DM in an exposure-dependent way. Large and well-designed trials are still needed to establish the efficacy and understand whether the effects were attributed to vibration, exercise, or a combination of both.
\end{abstract}

Keywords: type 2 diabetes mellitus; exercise; physical activity; whole body vibration; blood glucose; glycemic control.

PROSPERO: CRD42014010495.

\section{BULLET POINTS}

-WBV plus exercise slightly decrease fasting blood glucose in T2DM.

- Evidence of WBV effects on glycemic control improvement is limited in T2DM.

- Isolated effect of WBV on outcomes in T2DM still has not been investigated.

\section{HOW TO CITE THIS ARTICLE}

Robinson CC, Barreto RPG, Sbruzzi G, Plentz RDM. The effects of whole body vibration in patients with type 2 diabetes: a systematic review and meta-analysis of randomized controlled trials. Braz J Phys Ther. 2016 Jan-Feb; 20(1):4-14. http://dx.doi.org/10.1590/bjpt-rbf.2014.0133

\section{Introduction}

Physical activity plays an important role in prevention and control of type 2 diabetes mellitus (T2DM) and its related complications ${ }^{1}$. Both aerobic and resistance training improve insulin action and can assist with the management of blood glucose levels, lipids, blood pressure, cardiovascular risk, mortality, and quality of life; however, exercise must be undertaken regularly for continued benefits ${ }^{1,2}$. Nevertheless, most of people with T2DM are not active, mirroring the inertia of a lifetime of habits and motivational barriers such as lack of interest, lack of time, and depression ${ }^{3}$. In addition, physical disabilities and perceived discomfort when exercising are challenges to adherence to physical activity ${ }^{3,4}$.

Physical therapists are able to help people plan an individualized exercise program in order to maintain good blood glucose and achieve optimal weight ${ }^{5}$. To help people with diabetes improve their quality of life, physical therapists can intervene with physical

\footnotetext{
${ }^{1}$ Universidade Federal de Ciências da Saúde de Porto Alegre (UFCSPA), Porto Alegre, RS, Brazil

${ }^{2}$ Programa de Pós-graduação em Ciências da Saúde, UFCSPA, Porto Alegre, RS, Brazil

${ }^{3}$ Programa de Pós-graduação em Ciências da Reabilitação, UFCSPA, Porto Alegre, RS, Brazil

${ }^{4}$ Curso de Fisioterapia, Universidade Federal do Rio Grande do Sul (UFRGS), Porto Alegre, RS, Brazil

Received: Mar. 08, 2015 Revised: Apr. 04, 2015 Accepted: June 24, 2015
} 
treatment techniques such as manual or manipulative treatments, electrophysical agents, and mechanical agents $^{5,6}$.

Among the alternatives aimed to increase overall physical activity, whole body vibration (WBV) has been shown to be a new effective option in healthy subjects and individuals with several health conditions? It is assumed that vibration activates muscle spindles and evokes muscle contractions induced by a complex spinal and supraspinal neurophysiological mechanism known as tonic vibration reflex, allowing muscular activity enhancement even in static positions ${ }^{8}$.

Some systematic reviews ${ }^{9-14}$ summarized the effects of WBV in some outcomes of specific populations as follows: improvements in bone mineral density in postmenopausal women ${ }^{9}$; leg muscle strength ${ }^{10}$ and balance improvement in older individuals ${ }^{11}$; balance, gait, and proprioception improvement in individuals with neurological conditions such as Parkinson's disease, multiple sclerosis, and stroke ${ }^{12}$; pain intensity decrease and physical function enhancement in individuals with knee osteoarthritis ${ }^{13}$; and functional exercise capacity and quality of life improvement in people with chronic obstructive pulmonary disease $\mathrm{e}^{14}$. Furthermore, WBV requires significantly less time than conventional training and, therefore, reached a satisfactory compliance in previously inactive patients ${ }^{11}$.

Nevertheless, the effects of WBV in patients with T2DM were infrequently reported through a case report ${ }^{15}$ and acute $^{16}$, crossover $^{17}$, or pilot ${ }^{18,19}$ studies. In the last years, few randomized controlled trials were performed ${ }^{20,21}$ with conflicting results. To summarize the current evidence, we aimed carry out a systematic review of the effects of WBV intervention on the blood glucose levels, blood and physical cardiovascular risk factors, and physical and functional capacity of individuals with T2DM in comparison with a control or other intervention group.

\section{Method}

This systematic review was performed in accordance with the Cochrane Handbook for Systematic Reviews of Interventions ${ }^{22}$ and the recommendations of the Brazilian Journal of Physical Therapy tutorial ${ }^{23}$. The protocol of this systematic review was prospectively registered at PROSPERO under the identification CRD42014010495 and can be assessed online ${ }^{24}$.

\section{Data sources and searches}

Comprehensive literature searches were performed on the following electronic databases (from inception to June $1^{\text {st }}, 2015$ ): MEDLINE (accessed by PubMed), LILACS, Physiotherapy Evidence Database (PEDro), and Cochrane Central Register of Controlled Trials (Cochrane CENTRAL). The search terms included 'Whole body vibration', 'Diabetes' MeSH and synonyms, and a string of terms to optimize randomized controlled trial searches on PubMed ${ }^{25}$. In order to improve sensitivity, outcomes were not included in the search strategy. The references list of the articles identified in these searches were used as an additional source to identify other potentially eligible trials. The search strategy used on PubMed database can be fully assessed online ${ }^{26}$.

Randomized controlled trials were considered eligible if they addressed the effects of WBV on blood glucose levels, blood and physical cardiovascular risk factors, and physical and functional capacity in adult patients with T2DM, with a minimum of four weeks intervention and at least a control group not performing WBV. We considered as the primary outcome blood glucose levels, assessed by 12-hours fasting blood glucose (12-h FBG) or glycated hemoglobin (HbA1c). The secondary outcomes were blood and physical cardiovascular risk factors (blood cholesterol and triglycerides, atherogenic index, body mass index, body composition, weight, waist circumference, waist to hip ratio, blood pressure, or heart hate) and physical and functional capacity (maximal oxygen uptake, six-minute walk test (6MWT) distance, muscle strength, or static and dynamic postural balance). The exclusion criteria were studies that included individuals with stated diabetic complications (e.g. diabetic peripheral neuropathy, retinopathy, or nephropathy) and studies with an unreliable description of WBV.

\section{Study selection}

Two independent reviewers screened the titles and abstracts of all studies identified through the search strategies. A standard screening checklist based on the eligibility criteria was used for each study. Studies that did not meet the eligibility criteria, according to titles or abstracts, were excluded. The two independent reviewers retrieved full text versions of the remaining studies for a second review. There were no disagreements between reviewers.

\section{Data extraction and quality assessment}

Two reviewers independently extracted the data from the eligible studies by using a standardized data extraction form. The following data were extracted: authors; year of publication; number of individuals analyzed; gender; age; parameters of WBV and 
description of intervention; type of comparison; mean and standard deviation of pre and post assessments of each outcome available. From articles referred to the same participants, the article with the larger sample was considered and the article with the smaller sample was excluded if outcome measurements were duplicated. There were no disagreements between reviewers. HbA1c and 12-h FBG mean and standard deviation values were not available in one published study ${ }^{14}$, but the authors informed these estimates by email.

The studies were assessed regarding methodological quality and statistical reporting using the PEDro scale $^{27}$. When methodological quality assessment was not available on the PEDro database, two reviewers performed the ratings using the Brazilian Portuguese version of the PEDro scale ${ }^{27}$ items. In addition, the quality of each article was evaluated based on the recommendation of the International Society of Musculoskeletal and Neuronal Interactions (ISMNI) ${ }^{28}$ for reporting WBV intervention studies, consisting of 13 minimal reporting items about the WBV parameters and participant positioning. The instruments were rated independently by two reviewers. There were no disagreements between reviewers.

\section{Data synthesis and analysis}

After data extraction, if the outcome values could not be transformed into a common numeric scale for quantitative synthesis, a descriptive synthesis was performed. For quantitative synthesis, pooled-effect estimates were obtained by comparing the change from baseline to study end for intervention and control group. The procedures for estimation of missing data ${ }^{22}$ were performed to obtain the standard deviation difference. Results were presented as weighted mean difference (WMD) with their respective $95 \%$ confidence intervals (CI). Meta-analysis was performed using the random effects model. The statistical heterogeneity among studies was assessed using Cochran's Q test and the inconsistency $\mathrm{I}^{2}$ test, in which values above $25 \%$ and $50 \%$ were considered as indicatives of moderate and high heterogeneity, respectively. Sensitivity analysis was not possible given the number of available studies, therefore when $\mathrm{I}^{2}>25 \%$, meta-analysis was not considered. A p value lower than 0.05 was considered statistically significant. All analyses were conducted using Review Manager, version 5.2.

\section{Results}

\section{Description of studies}

The search strategy yielded 585 articles. From these, eight ${ }^{16,19-21,29-32}$ were considered as potentially relevant and retrieved for a detailed analysis. After full-text reading, four articles were excluded. As three $\operatorname{articles}^{21,31,32}$ referred to the same original study (clinical trial register: ACTRN12613000021774), they were considered as a single study. From this, two studies reporting outcomes on four different articles ${ }^{20,21,31,32}$ were included in this systematic review. Figure 1

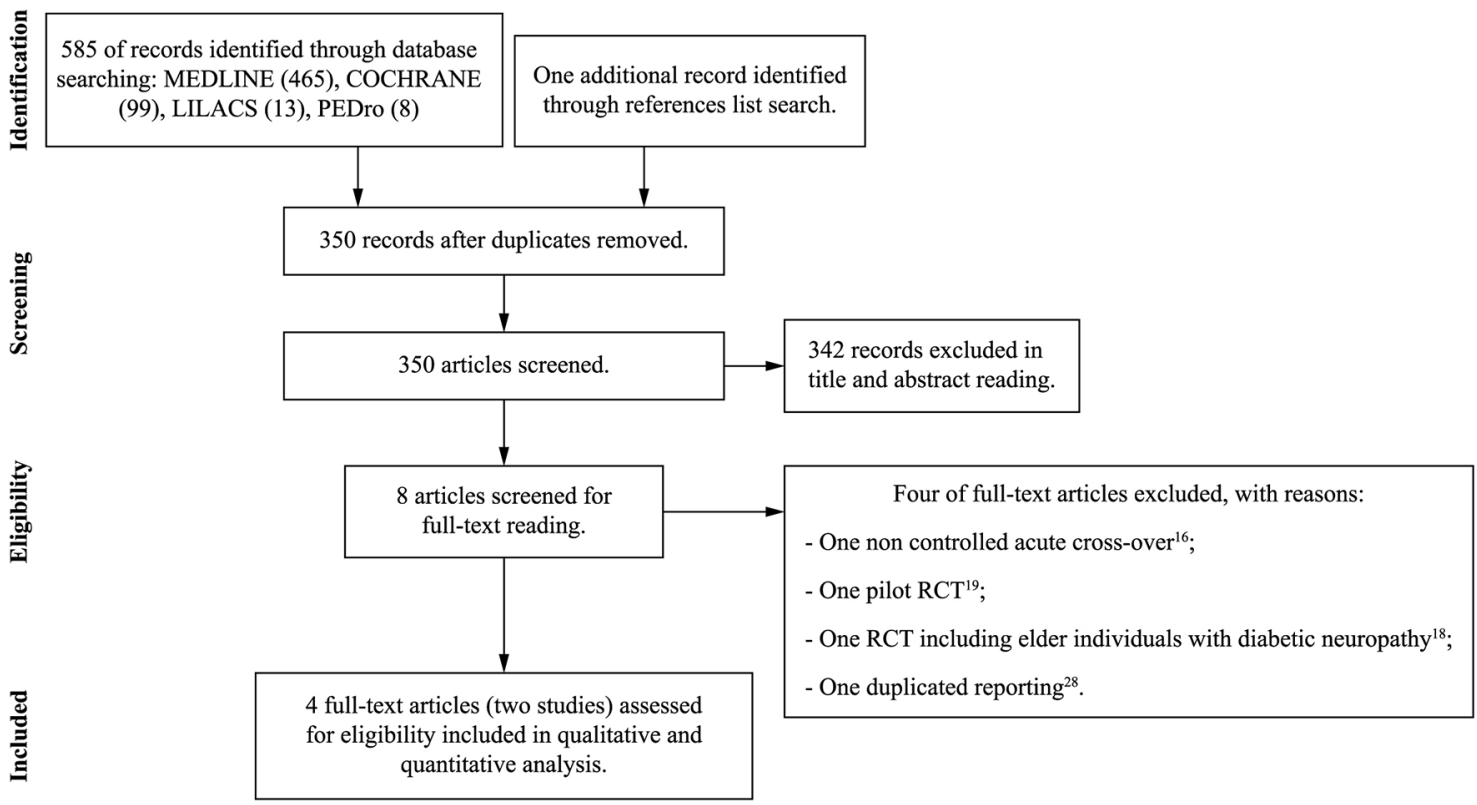

Figure 1. Flow diagram of studies included. 


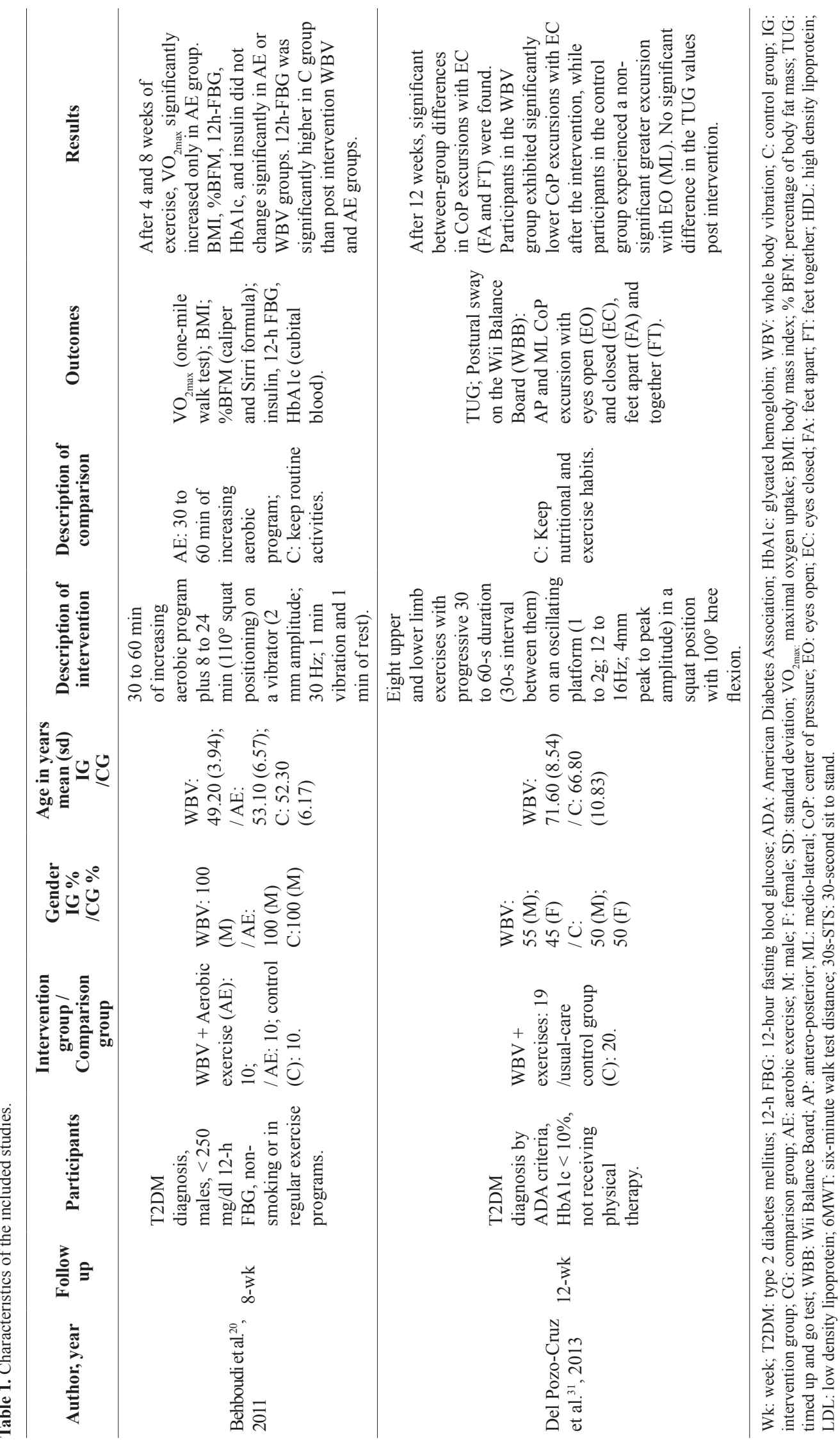




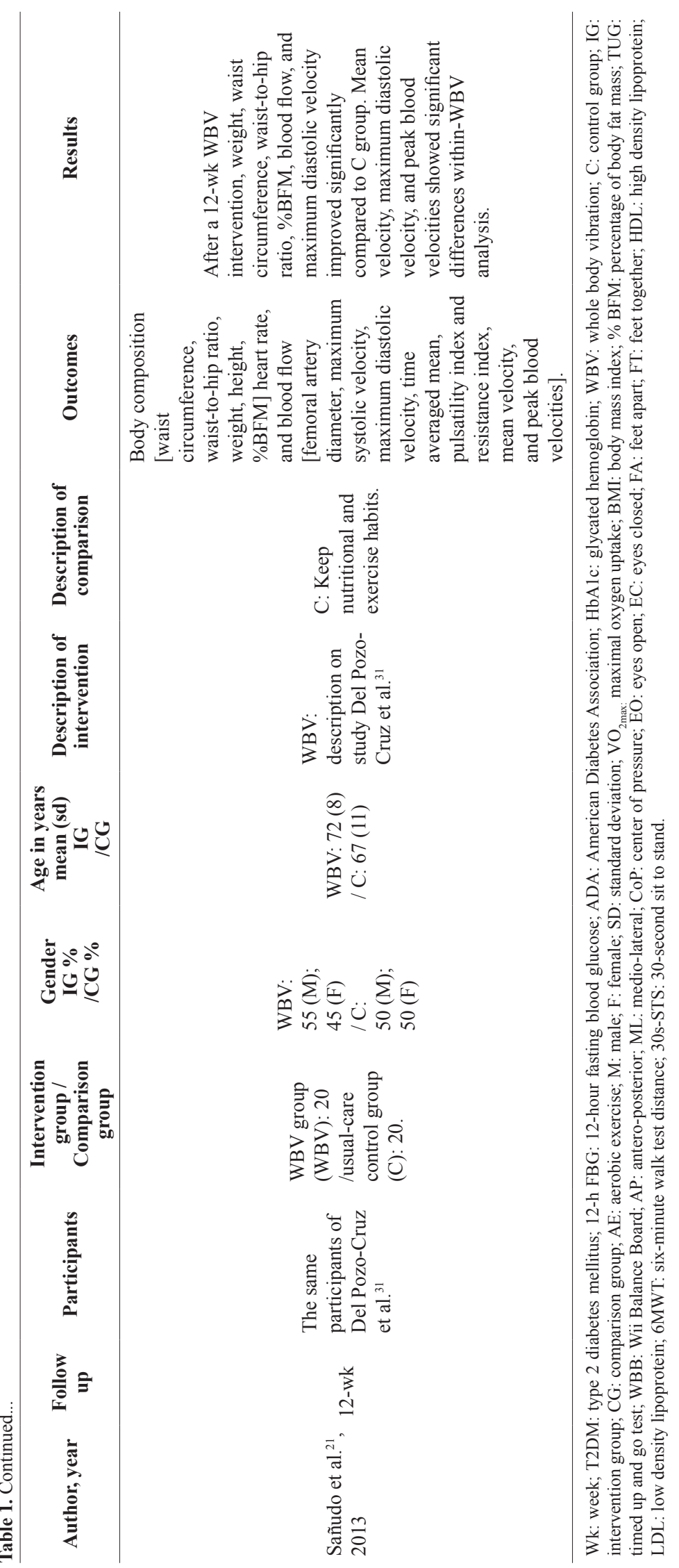




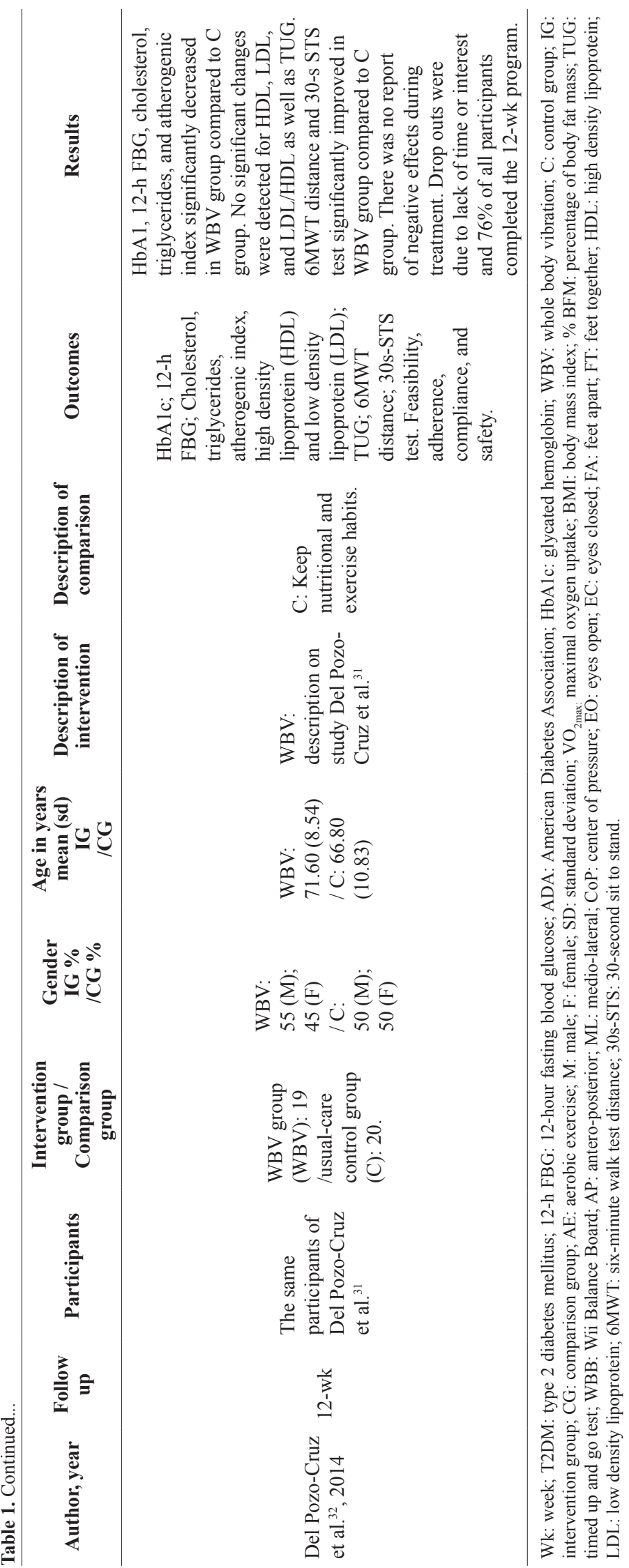


shows the flow diagram of the studies and Table 1 summarizes their characteristics.

A total of 70 participants with T2DM were assessed. The year of publication of the included studies ranged from 2011 to 2014. Both the studies included individuals with T2DM diagnosis and excluded those with established diabetes complications and $\mathrm{HbA} 1 \mathrm{c}>10 \%$ or fasting blood glucose $>250 \mathrm{ml} / \mathrm{dl}$. Age ranged from adult to elderly classification and only males were included by Behboudi et al. ${ }^{20}$ while the other study ${ }^{21,31,32}$ included both genders. All of the studies randomly allocated the individuals to a control group without additional intervention, keeping normal daily activities and medical instructions. In addition, Behboudi et al. ${ }^{20}$ randomly allocated individuals to a third group that performed an increasing aerobic exercise (AE) program only.

Regarding WBV intervention, both studies ${ }^{20,21,31,32}$ applied an intermittent exposure to WBV and acceleration and frequency parameters were very similar. Studies kept peak acceleration between 1 and $2 \mathrm{~g}$ (units of gravity; $1 \mathrm{~g}=1 \mathrm{~m} \cdot \mathrm{s}^{-2}$ ). In Behboudi et al. ${ }^{20}$, the peak acceleration was influenced mainly by higher vibration frequencies and lower amplitude, but in Sañudo et al. ${ }^{21}$ and Del Pozo-Cruz et al. ${ }^{31,32}$, higher amplitude and lower vibration frequencies determined the peak acceleration.
Both the studies proposed a thrice-weekly intervention of WBV with total session duration increasing progressively from 12 (8-16) to 14 (16-24) minutes. All of the studies designed protocols in which individuals stood on the vibrating platform in a 100 to $110^{\circ}$ squat position (considering total knee extension as $180^{\circ}$ ) and the vibratory stimulus was not isolated. Behboudi et al..$^{20}$ proposed $\mathrm{WBV}$ in addition to an increasing $\mathrm{AE}$ program $(\mathrm{WBV}+\mathrm{AE})$ with a follow-up after eight weeks. The study reported by Sañudo et al. ${ }^{21}$ and Del Pozo-Cruz et al. ${ }^{31,32}$ proposed a protocol of lower and upper limb exercises performed on the vibrating platform with a follow-up after 12 weeks.

No adverse effects were reported in any of the studies. Loss of follow-up occurred only in the assessment after 12 weeks ${ }^{21,31,32}$, in which five participants from the control group dropped out because of lack of interest. Six participants from the intervention group dropped out because of lack of time (five participants) and change of home address (one participant). Participants attended more than $75 \%$ of the sessions in both trials ${ }^{20,21,31,32}$.

Overall, the methodological quality assessed by the PEDro scale was low to moderate (Table 2). Table 3 shows the quality of each article based on the recommendation of the ISMNI ${ }^{28}$ for reporting WBV intervention studies.

Table 2. Methodological quality assessment by the Physiotherapy Evidence Database (PEDro) Scale.

\begin{tabular}{lcccccccccccc}
\hline \multicolumn{1}{c}{} & \multicolumn{10}{c}{ PEDro Scale Items } \\
\hline \multicolumn{1}{c}{ Author } & $\mathbf{1}$ & $\mathbf{2}$ & $\mathbf{3}$ & $\mathbf{4}$ & $\mathbf{5}$ & $\mathbf{6}$ & $\mathbf{7}$ & $\mathbf{8}$ & $\mathbf{9}$ & $\mathbf{1 0}$ & $\mathbf{1 1}$ & Score \\
\hline Behboudi et al. $^{20}$ & Yes & Yes & No & Yes & No & No & No & Yes & No & Yes & No & $4 / 10^{*}$ \\
Del Pozo-Cruz et al. ${ }^{31}$ & Yes & Yes & No & Yes & No & No & Yes & No & No & Yes & Yes & $5 / 10$ \\
Sañudo et al. ${ }^{21}$ & No & Yes & No & Yes & No & No & Yes & No & No & Yes & Yes & $5 / 10$ \\
Del Pozo-Cruz et al. ${ }^{32}$ & Yes & Yes & No & Yes & No & No & No & No & No & Yes & Yes & $4 / 10$
\end{tabular}

1: Eligibility criteria; 2: Random allocation; 3: Concealed allocation; 4: Baseline comparability; 5: Blind subjects; 6: Blind therapists; 7: blind assessors; 8: Adequate follow up; 9: "Intention-to-treat" analysis; 10: Between-group comparisons; 11: Point estimates and variability. Eligibility criteria item does not contribute to total score. *The methodological quality assessment was performed by the reviewers.

Table 3. Assessment of minimum items reported for whole body vibration interventions.

\begin{tabular}{|c|c|c|c|c|c|c|c|c|c|c|c|c|c|}
\hline \multirow[b]{2}{*}{ Author } & \multicolumn{13}{|c|}{ International Society of Musculoskeletal and Neuronal Interactions Items } \\
\hline & 1 & 2 & 3 & 4 & 5 & 6 & 7 & 8 & 9 & 10 & 11 & 12 & 13 \\
\hline Behboudi et al. ${ }^{20}$ & Yes & No & Yes & Unclear & No & No & No & Yes & No & No & No & Yes & Unclear \\
\hline Del Pozo-Cruz et al. ${ }^{31}$ & Yes & Yes & Yes & Yes & Yes & No & No & Yes & Yes & No & Yes & Yes & Yes \\
\hline Sañudo et al. ${ }^{21}$ & Yes & No & Yes & No & No & No & No & Yes & No & No & No & Yes & Yes \\
\hline Del Pozo-Cruz et al. ${ }^{32}$ & Yes & Yes & Yes & Yes & No & No & No & Yes & No & No & No & Yes & Yes \\
\hline
\end{tabular}

1: Brand name of vibration platform; 2: Type of vibration; 3: Vibration frequency; 4: Vibration amplitude; 5: Peak acceleration; 6: Accuracy of vibration parameter; 7: Evaluation of skidding of the feet; 8: Changes of vibration parameters; 9: Rationale for choosing vibration parameters; 10: Support devices during vibration exposure; 11: Type of footwear; 12: Body position; 13: Description of exercise. 


\section{Blood glucose levels}

For 12-h FBG, meta-analysis was performed and included data of two trials ${ }^{20,32}$ with a total of 59 patients (29 of which were on WBV). The comparison groups did not perform any intervention. There was an improvement in 12-h FBG by reduction in $25.7 \mathrm{ml} / \mathrm{dl}$ (95\% CI: -45.3 to $-6.1 ; \mathrm{I}^{2}: 19 \%$ ), favoring WBV intervention (Figure 2A). There was no additional effect $(\mathrm{p}=0.09)$ of WBV to an eight-week increasing AE program regarding 12-h FBG, but both the groups (WBV $+\mathrm{AE}$ and $\mathrm{AE}$ only) presented significantly lower 12-h FBG levels $(\mathrm{p}=0.02)$ than the control group ${ }^{20}$.

Regarding $\mathrm{HbA1c}$, a meta-analysis was not considered given an $\mathrm{I}^{2}$ of $80 \%$ between studies. After the 12-week program of upper and lower limb exercises performed on the vibrating platform, participants in the intervention group exhibited significantly lower levels of HbA1c $(p=0.002)$ at the time of follow-up when compared to the control group, with a mean difference of $-0.55 \%$ (95\% CI: -0.15 to -0.76$)^{32}$. The eight-week WBV+AE program was not sufficient to promote a significant difference in $\mathrm{HbAlc}$ levels compared to the control group. Furthermore, there was no additional effect of WBV on the eight-week AE program as no significant difference in $\mathrm{HbAlc}$ levels was found between $\mathrm{WBV}+\mathrm{AE}$ and $\mathrm{AE}$ only. Both intervention groups did not differ significantly from controls.

\section{Blood and physical cardiovascular risk factors}

Regarding secondary outcomes, a meta-analysis was only possible for Body Mass Index (BMI). Data of two studies ${ }^{20,31}$ with a total of 59 patients (29 of which were on $\mathrm{WBV}$ ) were included and comparison groups did not perform any intervention. A non-significant decrease of $0.67 \mathrm{Kg} . \mathrm{cm}^{-2}$ (95\% CI:-2.21 to $0.87 ; \mathrm{I}^{2}: 8 \%$ ) in BMI was observed (Figure 2B).

After the 12-week program of upper and lower exercises performed on the vibrating platform, a significant decrease $(\mathrm{p}<0.050)$ was found in cholesterol, triglycerides, atherogenic index ${ }^{32}$, weight, waist circumference, waist-to-hip ratio, and body fat percentage ${ }^{21}$ compared to the control group. However, no statistically significant changes were detected for high-density lipoprotein (LDL), low-density lipoprotein (LDL), or LDL/HDL ${ }^{32}$. After the eight-week $\mathrm{WBV}+\mathrm{AE}$ program, no significant differences in body fat percentage were found compared to the control group or compared to the $\mathrm{AE}$ group ${ }^{20}$.

\section{Physical and functional capacity}

Improvements $(p<0.05)$ were found in the $6 \mathrm{MWT}$ distance and muscle strength assessed by the 30 -second Sit-to-Stand (30s-STS) test after the 12-week WBV program with upper and lower limb exercises compared with the control group. Regarding static balance, the same comparison showed a significant decrease in center of pressure excursions with eyes

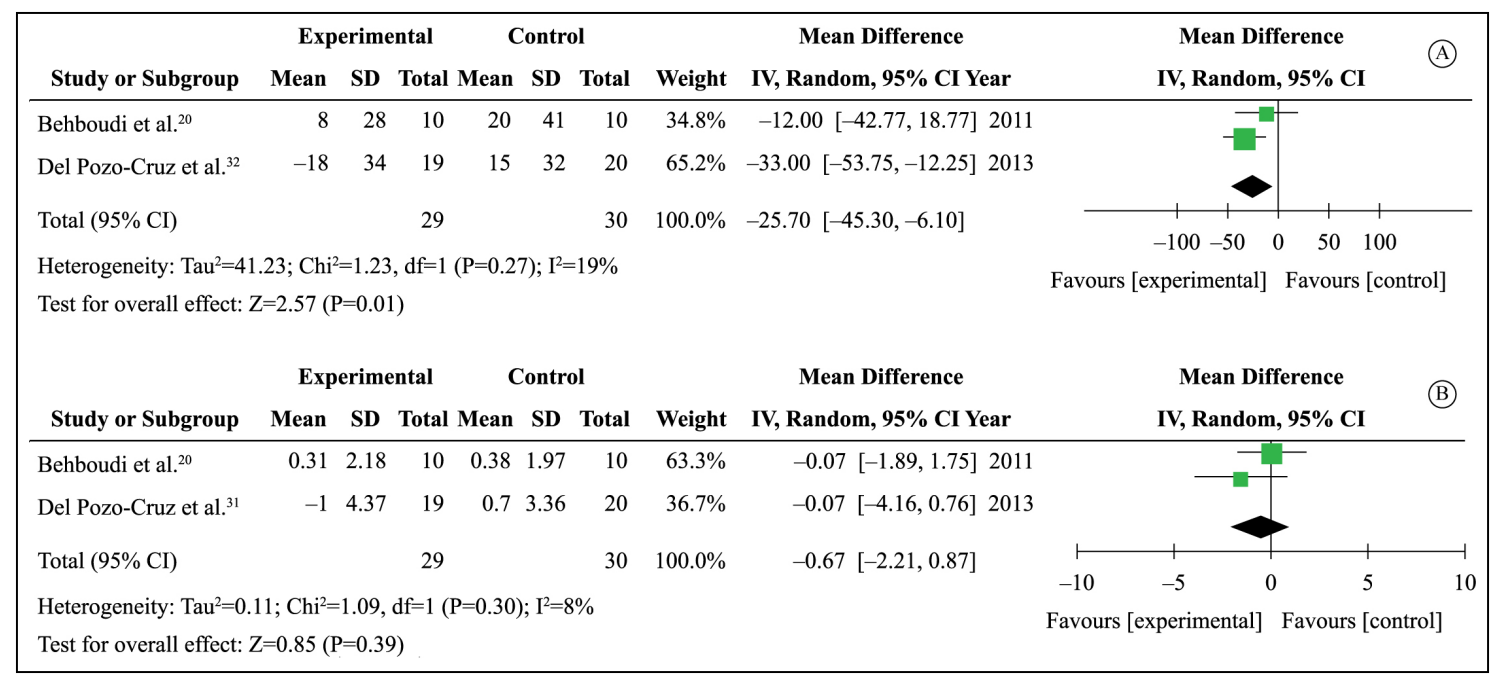

Figure 2. (A) The mean difference and 95\% confidence interval (CI) of 12-hour fasting blood glucose in $\mathrm{ml} / \mathrm{dl}$ for treatment with WBV (experimental) versus comparator (control); (B) Mean difference and $95 \%$ confidence interval (CI) of body mass index in $\mathrm{Kg} / \mathrm{cm}^{2}$ for treatment with WBV (experimental) versus comparator (control). 
closed (feet apart and together), but TUG time did not improve significantly ${ }^{32}$. Although maximal oxygen uptake increased significantly $(\mathrm{p}=0.01)$ after the eight-week WBV+AE and AE only programs, WBV had no additional effect on $\mathrm{AE}(\mathrm{p}=0.3)^{20}$.

\section{- Discussion}

\section{Summary of evidence}

It seems that the 12-week progressive intervention with WBV and exercise was sufficient for a statistically significant, but slight improvement in the 12-h FBG and $\mathrm{HbA} 1 \mathrm{c}$ of individuals with $\mathrm{T} 2 \mathrm{DM}$, in comparison with no intervention. Furthermore, the eight-week intervention improved 12-h FBG, but did not improve HbAlc.

Because erythrocytes are freely permeable to glucose, the level of $\mathrm{HbA} 1 \mathrm{c}$ in a blood sample provides a glycemic history of the previous 120 days, the average erythrocyte lifespan ${ }^{33}$. It is possible that a period of eight weeks was not enough to reach modifications in blood glucose profile, as no significant alterations were found in the $\mathrm{WBV}+\mathrm{AE}$ or $\mathrm{AE}$ only programs.

The effect size for $\mathrm{HbA1c}$ improvement discovered after the 12-week progressive intervention with WBV and exercise was close to the one found after aerobic or resistance training reported previously in two meta-analyses ${ }^{34,35}$. Although the vibratory stimulation was not isolated from exercises in the proposed interventions, session duration was considerably lower in the WBV studies (8 to 24 minutes) than in the aerobic or resistance training studies (40 to 75 minutes) ${ }^{34,35}$. This fact corroborates other studies that found similar results in WBV application compared conventional intervention, but in a shorter time of exposure ${ }^{36-38}$.

The meta-analysis for BMI found no significant decrease after the WBV interventions. According to Cochrane ${ }^{39}$, although WBV has gained popularity as a modality for weight loss, it does not have the ability to generate large energy expenditure to substitute conventional aerobic exercise. However, it had positive effects on blood flow ${ }^{32,40}$ that could indirectly improve associated diseases such as hypertension. In fact, this could be seen in some of the blood and physical markers of cardiovascular risk (cholesterol, triglycerides, atherogenic index, body weight, waist circumference, and waist-to-hip ratio) that improved after 12 weeks of progressive intervention with WBV combined with exercises ${ }^{32}$.

It seems that an eight-week WBV intervention was not enough to reach significant improvements in the aerobic capacity ${ }^{20}$ of patients with T2DM. In contrast, the 12-week progressive intervention with $\mathrm{WBV}$ and exercise improved aerobic capacity measured by the 6MWT distance, with similar values to those found in a multi-center study on fitness among healthy elderly subjects $^{41}$. The same improvement was found in lower limb strength measured through the $30 \mathrm{~s}-\mathrm{STS}$. It is possible that the time of exposure in patients with T2DM must be greater than that required for the non-diabetic population. For example, a previous meta-analysis found a significant beneficial effect of WBV on lower limb strength of elderly subjects with a treatment effect comparable to other forms of active exercises (e.g. resistance training) within six to 10 weeks $^{10}$.

\section{Limitations and conclusions}

This is the first systematic review to synthesize the effects of these outcomes in individuals with T2DM after WBV interventions. Analysis from data extraction of this systematic review was limited by the small number of available trials and duplicated articles. Furthermore, results from this systematic review must be interpreted with caution as most of the trials have some methodological limitations such as lack of concealed allocation and intention-to-treat analysis. Regarding the minimal items required for WBV intervention reproducibility, clear reporting is still necessary of the type of vibration, whether amplitude displacement was peak-to-peak, the peak acceleration, whether and how accuracy of vibration parameter were assessed, whether and how skidding of the feet were avoided, what was the rationale for choosing specific vibration parameters, whether and what support devices were used during vibration exposure and whether the type of footwear was controlled. Failing to report those items impairs protocol reproducibility as well as protocol comparison ${ }^{28}$.

Despite the slight beneficial effect of WBV intervention on glycemic control, a paramount outcome for T2DM management, caution is required in extrapolating this result to practice. First, a significant reduction in glycemic values was found in comparison with no intervention and WBV was not investigated alone, but in addition to exercise. Similar caution must be taken regarding blood markers and functional capacity. Even if WBV parameters were very similar between studies, the combined exercises differed between studies and follow-up was also distinct, which may have influenced pooled effects and heterogeneity. It is necessary to highlight that these implications should 
only be considered for patients with T2DM without reported complications or contraindications for WBV exposure as well as glycemic profile $<10 \%$ for $\mathrm{HbA} 1 \mathrm{C}$ or $<250 \mathrm{mg} / \mathrm{dl}$ for 12-h FBG. Furthermore, it seems that effectiveness of WBV is exposure-related as the 12-week intervention presented the better results.

Similarly to other studies that used WBV as an intervention in sedentary or elderly individuals ${ }^{10}$, there was good adherence and compliance in the 8-week and 12-week follow-up assessments. There was similar loss of follow-up in the intervention and control groups in the 12-week WBV program related to personal reasons ${ }^{21,31,32}$. Adverse effects, such as hypoglycemia, discomfort, and musculoskeletal injuries, are highly reported in studies performing exercise interventions ${ }^{34}$, however they were not reported in the studies included in this systematic review ${ }^{20,21,31,32}$.

WBV performed close to the parameters presented in the primary studies and combined with low-level exercises seems to be a safe, feasible, and less time-consuming intervention to help improve the glycemic control, cardiovascular risk markers, and functional capacity of individuals with T2DM in an exposure-dependent way compared to no intervention. However, given the methodological weaknesses of the primary studies and the heterogeneous protocols, confidence is limited on the decreasing effect of WBV on 12-h FBG. Further well-designed trials are still required to strengthen the current evidence and clarify whether the effect should be attributed to vibration, exercise, or the combination of both.

\section{References}

1. Colberg SR, Sigal RJ, Fernhall B, Regensteiner JG, Blissmer BJ, Rubin RR, et al. Exercise and type 2 diabetes. The American College of Sports Medicine and the American Diabetes Association: joint position statement. Diabetes Care. 2010;33(12):e147-67. http://dx.doi.org/10.2337/dc109990. PMid:21115758.

2. Hayashino Y, Jackson JL, Fukumori N, Nakamura F, Fukuhara S. Effects of supervised exercise on lipid profiles and blood pressure control in people with type 2 diabetes mellitus: a meta-analysis of randomized controlled trials. Diabetes Res Clin Pract. 2012;98(3):349-60. http://dx.doi.org/10.1016/j. diabres.2012.10.004. PMid:23116535.

3. Morrato EH, Hill JO, Wyatt HR, Ghushchyan V, Sullivan PW. Physical activity in U.S. adults with diabetes and at risk for developing diabetes. Diabetes Care. 2007;30(2):203-9. http://dx.doi.org/10.2337/dc06-1128. PMid:17259482.

4. Praet SFE, van Loon LJC. Exercise: the brittle cornerstone of type 2 diabetes treatment. Diabetologia. 2008;51(3):398-401. http://dx.doi.org/10.1007/s00125-007-0910-y. PMid:18183362.

5. Kalra S, Kalra B, Kumar N. Prevention and management of diabetes: the role of the physiotherapist. Diabetes Voice. 2007;52(3):12-4.
6. Andrade AD, Dean E. Aligning Physical Therapy practice with Brazil's leading Health priorities: a "call to action" in the $21^{\text {st }}$ century. Rev Bras Fisioter. 2008;12(4):260-7. http://dx.doi.org/10.1590/S1413-35552008000400003.

7. Cochrane DJ. Vibration exercise: the potential benefits. Int J Sports Med. 2011;32(2):75-99. http://dx.doi. org/10.1055/s-0030-1268010. PMid:21165804.

8. Zaidell LN, Mileva KN, Sumners DP, Bowtell JL. Experimental evidence of the tonic vibration reflex during whole-body vibration of the loaded and unloaded leg. PLoS One. 2013;8(12):e85247. http://dx.doi.org/10.1371/journal. pone.0085247. PMid:24386466.

9. Slatkovska L, Alibhai MH, Beyene J, Cheung AM. Effect of whole-body vibration on BMD: a systematic review and meta-analysis. Osteoporos Int. 2010;21(12):1969-80. http:// dx.doi.org/10.1007/s00198-010-1228-z. PMid:20407890.

10. Lau RW, Liao LR, Yu F, Teo T, Chung RC, Pang MY. The effects of whole body vibration therapy on bone mineral density and leg muscle strength in older adults: a systematic review and meta-analysis. Clin Rehabil. 2011;25(11):975-88. http://dx.doi.org/10.1177/0269215511405078. PMid:21849376.

11. Lam FM, Lau RW, Chung RC, Pang MY. The effect of whole body vibration on balance, mobility and falls in older adults: a systematic review and meta-analysis. Maturitas. 2012;72(3):206-13. http://dx.doi.org/10.1016/j. maturitas.2012.04.009. PMid:22609157.

12. Chanou K, Gerodimos V, Karatrantou K, Jamurtas A. Wholebody vibration and rehabilitation of chronic diseases: a review of the literature. J Sports Sci Med. 2012;11(2):187200. PMid:24149191.

13. Zafar H, Alghadir A, Anwer S, Al-Eisa E. Therapeutic effects of whole-body vibration training in knee osteoarthritis: a systematic review and meta-analysis. Arch Phys Med Rehabil. 2015;96(8):1525-32. http://dx.doi.org/10.1016/j. apmr.2015.03.010. PMid:25827655.

14. Yang X, Zhou Y, Wang P, He C, He H. Effects of whole body vibration on pulmonary function, functional exercise capacity and quality of life in people with chronic obstructive pulmonary disease: a systematic review. Clin Rehabil. 2015;2:0269215515589202. PMid:26038611.

15. Hong J, Barnes M, Kessler N. Case study: use of vibration therapy in the treatment of diabetic peripheral small fiber neuropathy. J Bodyw Mov Ther. 2013;17(2):235-8. http:// dx.doi.org/10.1016/j.jbmt.2012.08.007. PMid:23561872.

16. Yoosefinejad AK, Shadmehr A, Olyaei G, Talebian S, Bagheri $\mathrm{H}$. The effectiveness of a single session of whole-body vibration in improving the balance and the strength in type 2 diabetic patients with mild to moderate degree of peripheral neuropathy: a pilot study. J Bodyw Mov Ther. 2014;18(1):82-6. http://dx.doi.org/10.1016/j.jbmt.2013.10.007. PMid:24411154.

17. Johnson PK, Feland JB, Johnson AW, Mack GW, Mitchell UH Effect of whole body vibration on skin blood flow and nitric oxide production. J Diabetes Sci Technol. 2014;8(4):889-94. http://dx.doi.org/10.1177/1932296814536289. PMid:24876449.

18. Kessler NJ, Hong J. Whole body vibration therapy for painful diabetic peripheral neuropathy: a pilot study. J Bodyw Mov Ther. 2013;17(4):518-22. http://dx.doi.org/10.1016/j. jbmt.2013.03.001. PMid:24139013.

19. Baum K, Votteler T, Schiab J. Efficiency of vibration exercise for glycemic control in type 2 diabetes patients. Int J Med Sci. 2007;4(3):159-63. http://dx.doi.org/10.7150/ ijms.4.159. PMid:17554399.

20. Behboudi L, Azarbayjani MA, Aghaalinejad H, Salavati M. Effects of aerobic exercise and whole body vibration on 
glycaemia control in type 2 diabetic males. Asian J Sports Med. 2011;2(2):83-90. PMid:22375223.

21. Sañudo B, Alfonso-Rosa R, Pozo-Cruz BD, Pozo-Cruz JD, Galiano D, Figueroa A. Whole body vibration training improves leg blood flow and adiposity in patients with type 2 diabetes mellitus. Eur J Appl Physiol. 2013;113(9):2245-52. http://dx.doi.org/10.1007/s00421-013-2654-3. PMid:23657766.

22. Higgins JPT, Green S, editors. Cochrane Handbook for Systematic Reviews of Interventions Version 5.1.0 [updated March 2011]. London: The Cochrane Collaboration; 2011 [cited 2015 March 15]. Available from: www.cochranehandbook.org.

23. Mancini MC, Cardoso JR, Sampaio RS, Costa LCM, Cabral CMN, Costa LOP. Tutorial for writing systematic reviews for the Brazilian Journal of Physical Therapy (BJPT). Braz J Phys Ther. 2014;18(6):471-80. http://dx.doi.org/10.1590/ bjpt-rbf.2014.0077. PMid:25590440.

24. Robinson CC, Barreto RPG, Plentz RDM, Sbrizzi G. Effects of whole body vibration in individuals with type 2 diabetes mellitus: a systematic review and meta-analysis of randomized clinical trials. Heslington: Centre for Reviews and Dissemination, University of York. PROSPERO 2014:CRD42014010495 [cited 015 March 15]. Available from: http://www.crd.york.ac.uk/ PROSPERO/display_record.asp?ID=CRD42014010495\#. VBTsCpRdWSo.

25. Robinson KA, Dickersin K. Development of a highly sensitive search strategy for the retrieval of reports of controlled trials using Pub Med. Int J Epidemiol. 2002;31(1):150-3. http://dx.doi.org/10.1093/ije/31.1.150. PMid:11914311.

26. University of York. Centre for Reviews and Dissemination - CRD. Heslington [cited 2015 March 15]. Available from: http://www.crd.york.ac.uk/PROSPEROFILES/10495 STRATEGY_20140602.pdf.

27. Shiwa SR, Costa LO, Costa LCM, Moseley A, Hespanhol Junior LC, Venâncio R, et al. Reproducibility of the Portuguese version of the PEDro Scale. Cad Saude Publica. 2011;27(10):2063-8. http://dx.doi.org/10.1590/S0102311X2011001000019. PMid:22031210.

28. Rauch F, Sievanen H, Boonen S, Cardinale M, Degens $\mathrm{H}$, Felsenberg D, et al. Reporting whole body vibration intervention studies: recommendations of the International Society of Musculoskeletal and Neuronal Interactions. J Musculoskelet Neuronal Interact. 2010;10(3):193-8. PMid:20811143.

29. Lee K, Lee S, Song C. Whole-body vibration training improves balance, muscle strength and glycosylated hemoglobin in elderly patients with diabetic neuropathy. Tohoku J Exp Med. 2013;231(4):305-14. http://dx.doi. org/10.1620/tjem.231.305. PMid:24334483.

30. Azarbayjani MA, Behboudi L, Aghaalinejad H, Salavati M. Comparative effects of aerobic training and whole body vibration on plasma adiponectin and insulin resistance in type 2 diabetic men. Ann Biol Res. 2011;2(5):671-80.

31. Del Pozo-Cruz J, Alfonso-Rosa RM, Ugia JL, McVeigh JG, Pozo-Cruz BD, Sañudo B. A primary care based randomized controlled trial of 12-week whole-body vibration for balance improvement in type 2 diabetes mellitus. Arch Phys Med Rehabil. 2013;94(11):2112-8. http://dx.doi.org/10.1016/j. apmr.2013.05.030. PMid:23811317.
32. Del Pozo-Cruz B, Alfonso-Rosa RM, Pozo-Cruz JD, Sañudo B, Rogers ME. Effects of a 12-wk whole-body vibration based intervention to improve type 2 diabetes. Maturitas. 2014;77(1):52-8. http://dx.doi.org/10.1016/j. maturitas.2013.09.005. PMid:24094494.

33. Goldstein DE, Little RR, Lorenz RA, Malone JI, Nathan $\mathrm{DM}$, Peterson CM, et al. Tests of glycemia in diabetes. Diabetes Care. 2004;27(7):1761-73. http://dx.doi.org/10.2337/ diacare.27.7.1761. PMid:15220264.

34. Umpierre D, Ribeiro PA, Schaan BD, Ribeiro JP. Volume of supervised exercise training impacts glycaemic control in patients with type 2 diabetes: a systematic review with meta-regression analysis. Diabetologia. 2013;56(2):242-51. http://dx.doi.org/10.1007/s00125-012-2774-z. PMid:23160642.

35. Chudyk A, Petrella R. Effects of exercise on cardiovascular risk factors in type 2 diabetes. Diabetes Care. 2011;34(5):122837. http://dx.doi.org/10.2337/dc10-1881. PMid:21525503.

36. Delecluse C, Roelants M, Verschueren S. Strength increase after whole-body vibration compared with resistance training. Med Sci Sports Exerc. 2003;35(6):1033-41. http://dx.doi. org/10.1249/01.MSS.0000069752.96438.B0. PMid:12783053.

37. Roelants M, Delecluse C, Goris M, Verschueren S. Effects of 24 weeks of whole body vibration training on body composition and muscle strength in untrained females. Int J Sports Med. 2004;25(1):1-5. http://dx.doi.org/10.1055/s-2003-45238. PMid:14750005.

38. Bogaerts A, Delecluse C, Claessens AL, Coudyzer W, Boonen $\mathrm{S}$, Verschueren SM. Impact of whole-body vibration training versus fitness training on muscle strength and muscle mass in older men: a 1-year randomized controlled trial. J Gerontol A Biol Sci Med Sci. 2007;62(6):630-5. http:// dx.doi.org/10.1093/gerona/62.6.630. PMid:17595419.

39. Cochrane DJ. Is vibration exercise a useful addition to a weight management program? Scand J Med Sci Sports. 2012;22(6):705-13. http://dx.doi.org/10.1111/j.16000838.2011.01411.x. PMid:22092513.

40. Kerschan-Schindl K, Grampp S, Henk C, Resch H, Preisinger E, Fialka-Moser V, et al. Whole-body vibration exercise leads to alterations in muscle blood volume. Clin Physiol. 2001;21(3):377-82. http://dx.doi.org/10.1046/j.13652281.2001.00335.x. PMid:11380538.

41. Pedrero-Chamizo R, Gómez-Cabello A, Delgado S, RodríguezLlarena S, Rodríguez-Marroyo JA, Cabanillas E, et al. Physical fitness levels among independent non-institutionalized Spanish elderly: the elderly EXERNET multi-center study. Arch Gerontol Geriatr. 2012;55(2):406-16. http://dx.doi org/10.1016/j.archger.2012.02.004. PMid:22424779.

\section{Correspondence}

\section{Caroline Cabral Robinson}

Universidade Federal de Ciências da Saúde de Porto Alegre

Rua Sarmento Leite, 245

CEP 90050-170, Porto Alegre, RS, Brazil

e-mail: carollinerobinson@gmail.com 